\title{
Metabolomic phenotyping of a cloned pig model
}

\author{
Morten R Clausen ${ }^{1 *}$, Kirstine L Christensen ${ }^{2}$, Mette S Hedemann ${ }^{2}$, Ying Liư ${ }^{3}$, Stig Purup ${ }^{2}$, Mette Schmidt ${ }^{4}$, \\ Henrik Callesen ${ }^{3}$, Jan Stagsted ${ }^{5}$ and Hanne C Bertram ${ }^{1}$
}

\begin{abstract}
Background: Pigs are widely used as models for human physiological changes in intervention studies, because of the close resemblance between human and porcine physiology and the high degree of experimental control when using an animal model. Cloned animals have, in principle, identical genotypes and possibly also phenotypes and this offer an extra level of experimental control which could possibly make them a desirable tool for intervention studies. Therefore, in the present study, we address how phenotype and phenotypic variation is affected by cloning, through comparison of cloned pigs and normal outbred pigs.

Results: The metabolic phenotype of cloned pigs $(n=5)$ was for the first time elucidated by nuclear magnetic resonance (NMR)-based metabolomic analysis of multiple bio-fluids including plasma, bile and urine. The metabolic phenotype of the cloned pigs was compared with normal outbred pigs $(n=6)$ by multivariate data analysis, which revealed differences in the metabolic phenotypes. Plasma lactate was higher for cloned vs control pigs, while multiple metabolites were altered in the bile. However a lower inter-individual variability for cloned pigs compared with control pigs could not be established.

Conclusions: From the present study we conclude that cloned and normal outbred pigs are phenotypically different. However, it cannot be concluded that the use of cloned animals will reduce the inter-individual variation in intervention studies, though this is based on a limited number of animals.
\end{abstract}

\section{Background}

Use of animal models in research related to human health and nutrition is common practice for example in dietary intervention studies. There are several reasons for using animal models. Firstly, the access to several bio-fluids and organs is possible. Secondly, it is easier to control animals than humans and to secure compliance to the experimental diet. This should lead to smaller inter-individual differences which are necessary for showing effects of the compound/diet under investigation. For this reason we hypothesize that a cloned animal model would be beneficial for intervention studies, as they are expected to provide a more controlled and repeatable experimental system that requires fewer animals compared with outbred lines.

In particular, the pig has become a widely used model, since pigs from a nutritional aspect are comparable to humans [1,2], and their lipoprotein profile and

\footnotetext{
* Correspondence: MortenR.Clausen@agrsci.dk

'Department of Food Science, Science and Technology, Aarhus University, Aarslev, Denmark

Full list of author information is available at the end of the article
}

metabolism are similar to that of humans [3-6]. Recently, a cloned pig model was used as a model for studying atherosclerosis [7]. However, the use of cloned animals in nutrition studies is still in its opening stage, and a more comprehensive elucidation of the usefulness of a cloned pig model in these types of studies is needed. Cloned pigs will have identical DNA sequences and in principle identical phenotypes. However, in the cloning process, single somatic cell nuclei are introduced into each their enucleated oocyte containing mitochondrial DNA, so a small subset of mitochondrial proteins will be of maternal origin [8]. Additionally, the somatic cell nuclei may have different epigenetic constitution, i.e. although the DNA sequences are identical, the methylation degree may vary between nuclei which could lead to differences in expression of certain genes and therefore to variable phenotypes. Consequently, the phenotypic variation of cloned pigs is so far unknown, and there is only limited data available in the literature $[9,10]$.

The phenotype, i.e. an individual's observable traits, is expressed in the metabolome. Proton nuclear magnetic 
resonance $\left({ }^{1} \mathrm{H}\right.$ NMR $)$ spectroscopy is probably the most widely applied technique for studying the metabolome based on bio-fluids, and has been used for metabolic phenotyping of humans [11]. The porcine metabolome has also been subject to investigations, and the potential of NMR-based metabolomics for elucidating the biochemical effects of dietary components such as rye versus wheat fibers [12,13], and arginine supplementation [14] as well as for studying the impact of birth weight on the plasma metabolome has been established [15]. However, no metabolomic investigations have so far been reported on cloned pigs. The importance of such a characterization is further underlined by the widespread use of pigs as a model in studies of cardiovascular disease, diabetes, and the metabolic syndrome [2], since the usefulness of such a model must rely on similarities in phenotype and in response to experimental treatments.

Therefore the aim of the present study was to elucidate the phenotype of a cloned pig model by characterization of multiple bio-fluids (urine, plasma and bile) using NMR-based metabolomics by comparison with outbred control pigs.

\section{Results}

\section{Multivariate data analysis of bio-fluids}

Representative ${ }^{1} \mathrm{H}$ NMR spectra obtained for plasma, urine and bile are shown in Figure 1 (also see Additional File 1 and Additional file 2 for all data files). The NMR spectra were assigned by comparison with established libraries reported in the literature [16], the Human Metabolome Data Base (HMDB) [17], by comparison with previous studies $[18,19]$, and with pure standards. Potential differences in the NMR metabolite profiles between cloned pigs and control pigs were investigated using PCA. For plasma and bile a tendency for grouping of cloned pigs and control pigs was observed, whereas for urine no grouping was observed (Figures 2, 3 and 4). Furthermore, while the cross-validated predictive ability of the PCA models was good for plasma and bile, the cross-validated predictive ability was poor for the PCA model obtained on urine samples (Table 1 and Figure 2), and interpretation of these data would probably require a larger set of samples. For bile and plasma, PCA resulted in differentiation between cloned and control pigs irrespective of normalization. Classification of plasma, however, was due to a global difference in metabolite concentrations covering all resonances. No explanation could be elucidated for this difference, and thus, this model did not reveal any information on metabolic differences between the two groups. For bile, performances of the normalized and non-normalized models were similar (Table 1), and inspection of loadings showed that they were superimposable, thus leading to the same conclusions (not shown). Accordingly, for data in the present study normalization had no effect on bile samples, whereas normalization of plasma data had a prominent effect. All multivariate data analysis is therefore based on normalized data.

\section{Bile}

Loadings from PCA revealed that for bile, multiple signals in the NMR spectra contributed to the differentiation of cloned pigs and control pigs (Figure 3). Bile from cloned pigs was characterised by higher intensities of signals assigned to choline $\left(-\mathrm{N}-\left(\mathrm{CH}_{3}\right)_{3}, 3.22 \mathrm{ppm}\right)$, phosphatidyl choline (PC) glycerol moiety (4.2 - 4.5 ppm), and $\left.\mathrm{PC}\left(-\mathrm{CH}_{2}-\mathrm{N} 3.68 \mathrm{ppm}\right)\right)$. Unsaturated lipids, probably also related to phospholipids, and total lipids, were also more abundant in bile from cloned pigs. Signals from conjugated cholate and conjugated chenodeoxy cholate $(7.72-8.05$ ppm, 3.72 ppm; 3.56 ppm; $3.07 \mathrm{ppm})$ as well as their un-conjugated forms (3.46 ppm; $3.86 \mathrm{ppm}$ ) were consistently more abundant in bile from control pigs compared with cloned pigs.

\section{Plasma}

The PCA model of the NMR spectra of plasma samples included samples from all sampling days, thus including 9 - 11 samples of plasma from each individual pig. Manual inspection of PC1 to PC4 of the obtained model did not reveal any clustering according to sampling date, and this aspect was not investigated further. As shown in Figure 4, a clustering between cloned pigs and control pigs along PC2 could be observed and the loadings for this principal component indicates that this is mainly due to differences in lactate concentrations $(1.33 \mathrm{ppm}$ and $4.11 \mathrm{ppm}$ ) and to a minor extent signals from lipids, and a number of amino acids. Since multiple sampling was carried out an O-PLS-DA model could be constructed and cross-validated without risk of over-fitting data. This analysis confirmed that the difference between cloned pigs and control pigs should be ascribed to a higher concentration of lactate in plasma from cloned pigs compared with control pigs (Figure 5a and $5 b)$. The constructed S-plot (Figure $5 \mathrm{~b}$ ) revealed that also alanine (1.48 ppm), threonine (1.42 ppm), and glutamate $(2.45 \mathrm{ppm})$ were more abundant in plasma from cloned pigs, whereas in plasma from control pigs signals from lipoproteins (0.80 ppm; $1.20 \mathrm{ppm} ; 2.00 \mathrm{ppm} ; 5.25$ $\mathrm{ppm})$, creatine (3.03 ppm; $3.92 \mathrm{ppm})$, and choline (3.19 ppm) were most abundant. Relative integrals of these metabolites are presented in Table 2 and confirm these conclusions, and indicate that lactate and creatine are the most important discriminatory compounds.

\section{Effect of cloning on inter-individual variation}

The most intense signals were integrated, and the variances were calculated for each integral, in order to 

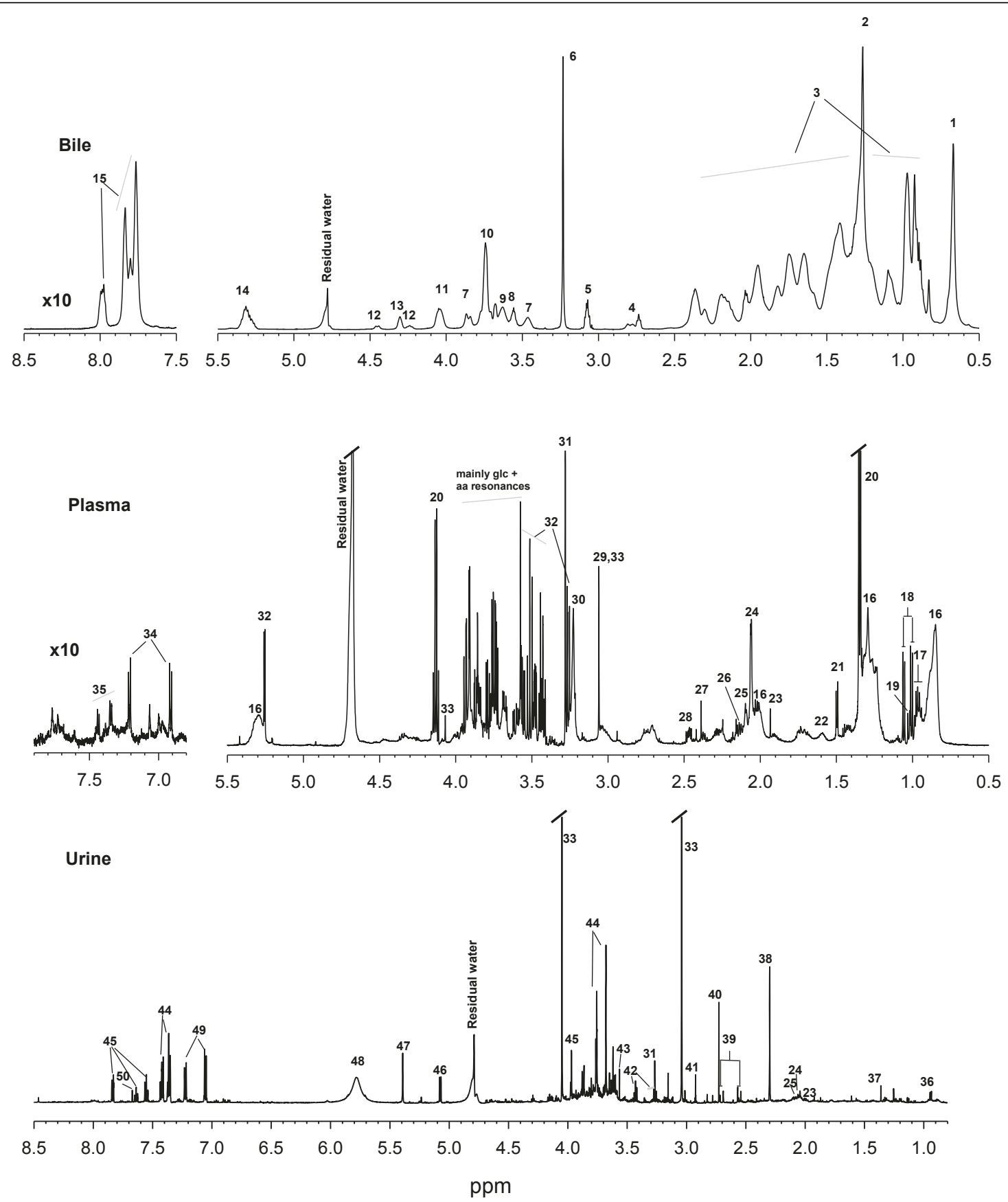

Figure 1 NMR spectra and assignments. Representative ${ }^{1} H$ NMR spectra of bile, plasma and urine obtained from a cloned pig. All spectra were acquired on a $600 \mathrm{MHz}$ spectrometer. Less abundant amino acids (Glu, Gln, Lys, and Arg) with complex spectra could not be unambiguously assigned in the spectral region $1.6-2.6$ ppm in urine. Assignments: 1, bile acids, cholesterol; $\mathbf{2}$, lipids (- $\mathrm{CH}_{2}$-); $\mathbf{3}$, bile acids cholesterol, lipids; $\mathbf{4}$, bisallyllic protons; $\mathbf{5}$, conjugated taurine; $\mathbf{6}$, choline or phosphatidylcholine (PC) $\left(-\mathrm{N}-\left(\mathrm{CH}_{3}\right)_{3}\right) ; \mathbf{7}$, glycine/taurine conjugated cholate (CA), glycine/taurine conjugated chenodeoxy cholate (CDCA); 8 , Conjugated deoxycholate $(\mathrm{H}-3 \beta)$, conjugated taurine $(\mathrm{H}-25) ; \mathbf{9}, \mathrm{PC}\left(\mathrm{CH} \mathbf{2}^{-}\right.$ $\mathrm{N}) ; \mathbf{1 0}$, Conjugated glycine (H-25); 11, conjugated cholate and deoxycholate, $\mathrm{PC}$-glycerol (3-CH2); 12, PC-glycerol (1-CH); 13, PC (-O-CH2-); 14, CH $=\mathrm{CH}$, cholesterol (6-CH), PC-glycerol (2-CH); 15; Conj bile acids (-NH-); 16, LDLNLDL; 17, leucine; 18, valine; 19, isoleucine; 20 , lactate; $\mathbf{2 1}$

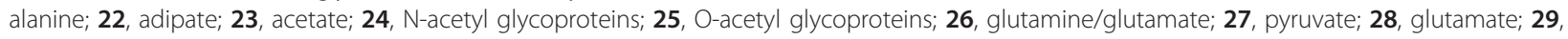
creatine; $\mathbf{3 0}$, choline; $\mathbf{3 1}$, trimethylamine-N-oxide (TMAO); $\mathbf{3 2}$, glucose; $\mathbf{3 3}$, creatinine; $\mathbf{3 4}$, tyrosine; $\mathbf{3 5}$, phenylalanine; $\mathbf{3 6}$, isovaleraldehyde; $\mathbf{3 7}, \alpha-$ hydroxyisobutyrate; $\mathbf{3 8}$, unknown; $\mathbf{3 9}$, citrate; $\mathbf{4 0}$, dimethylamine; $\mathbf{4 1}$, trimethyl amine; $\mathbf{4 2}$, taurine; $\mathbf{4 3}$, glycine; $\mathbf{4 4}$, phenylacetylglycine; $\mathbf{4 5}$, hippurate; $\mathbf{4 6}$, unknown; $\mathbf{4 7}$, alantoin; $\mathbf{4 8}$, urea; $\mathbf{4 9}$, unknown; $\mathbf{5 0}$, guanine. For further information about identified metabolites, please refer to Additional file 1 


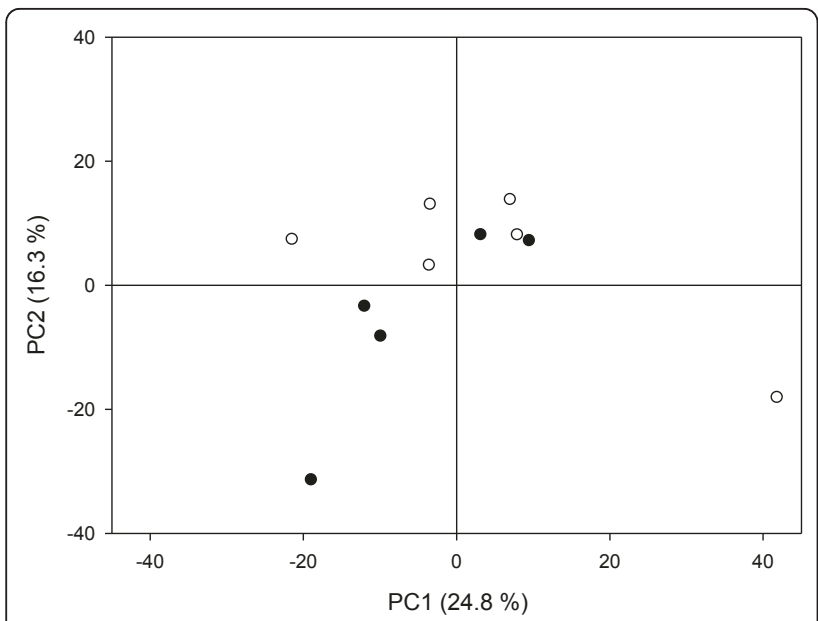

Figure 2 Urine PCA. PCA score plots of urine samples from cloned pigs (closed symbols) and control pigs (open symbols). $n=11$. Cumulated explained variance, $R^{2} X=0.57$, Cumulated cross validated explained variance, $Q^{2}($ cum $)=-0.17$ for the three component model made from normalized data.

elucidate the inter-individual variation for the cloned and control pigs. The ratios between the variances for cloned and for control pigs were then determined. These ratios provide a measure that can easily establish which group has the largest variation, as a value larger than one reflects a higher variation for cloned pigs than

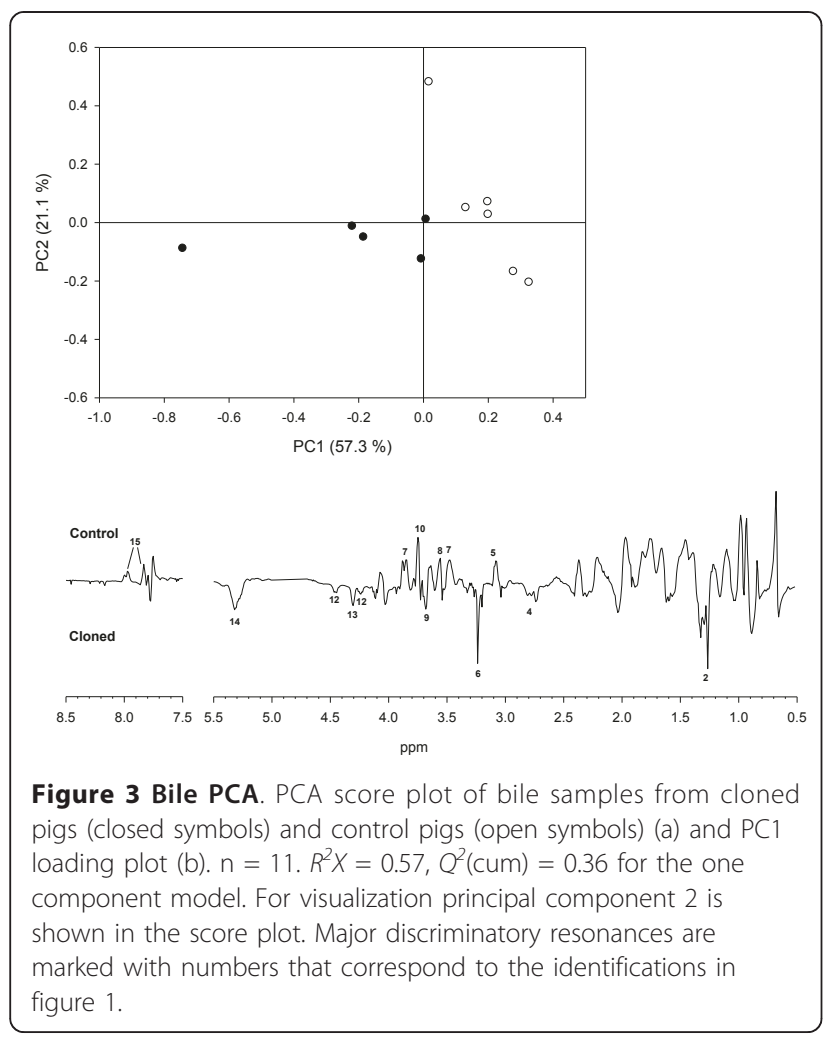

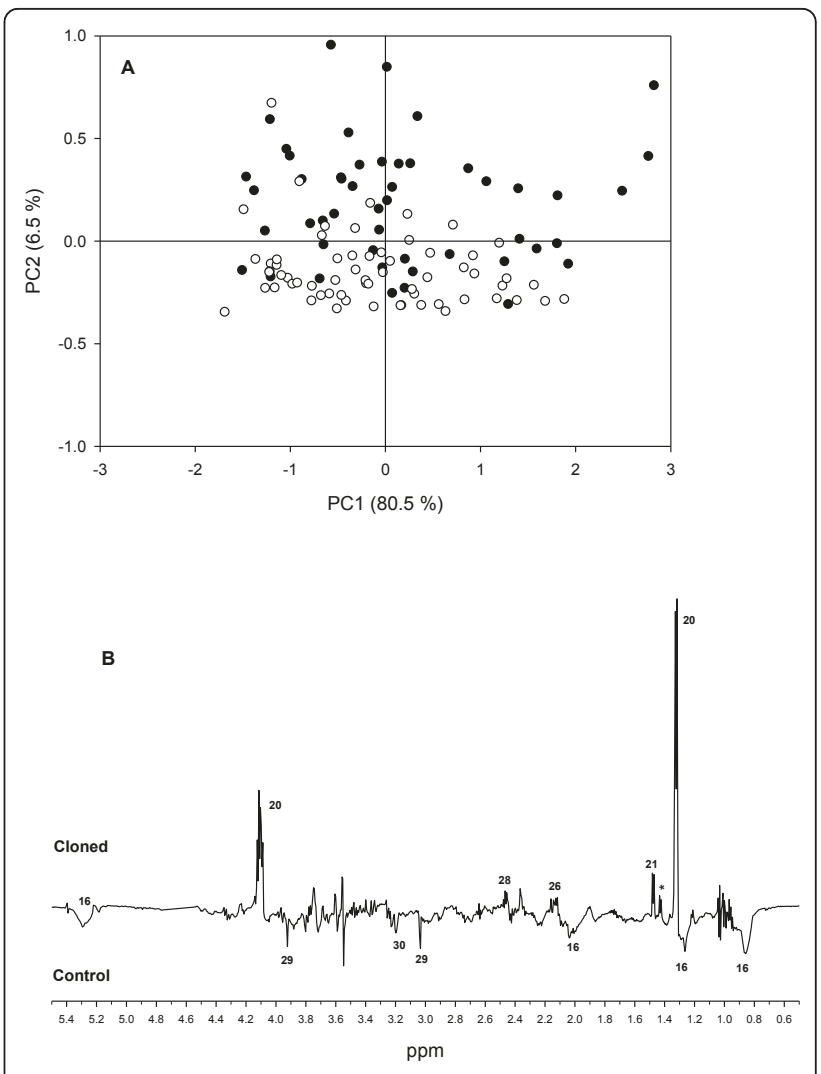

Figure 4 Plasma PCA. PCA score plot of plasma samples from cloned pigs (closed symbols) and control pigs (open symbols) (a) and PC2 loading plot (b). $\mathrm{n}=112 . R^{2} X=0.97, Q^{2}(\mathrm{cum})=0.74$ for the 10 component model. Major discriminatory resonances are marked with numbers that correspond to the identifications in figure 1. Asterisk denotes presumed threonine, which could not be resolved in the 1-D NMR spectrum.

for control pigs, while a value smaller than one reflects a lower variation for cloned pigs than for control pigs. Only well-resolved peaks were used for this analysis, and consequently 10,7 , and 15 signals were integrated in bile, plasma and urine respectively (Table 3 and Additional file 3). Only three significant differences in

Table 1 PCA models for NMR spectral data

\begin{tabular}{llllll}
\hline $\begin{array}{l}\text { Bio- } \\
\text { fluid }\end{array}$ & Normalization & $\mathbf{n}$ & $\begin{array}{l}\text { Number of } \\
\text { components }\end{array}$ & $\mathbf{R}^{2} \mathbf{X}$ & $\begin{array}{l}\mathbf{Q}^{2} \\
\text { (cum) }\end{array}$ \\
\hline Bile & + & 11 & 1 & 0.57 & 0.36 \\
Bile & $-{ }^{a}$ & 11 & 2 & 0.40 & 0.37 \\
\hline Urine & + & 11 & 3 & 0.57 & -0.17 \\
Urine & - & 11 & 2 & 0.39 & 0.12 \\
\hline Plasma & + & 110 & 10 & 0.97 & 0.74 \\
Plasma & - & 110 & 10 & 0.95 & 0.91 \\
\hline
\end{tabular}

Summary of PCA models of all biofluids, with and without normalization ${ }^{\mathrm{a}}$ First component was used to describe the variation induced by one outlier. This outlier was included in the analysis, since the second component, which described the difference between groups, was not affected by it. 

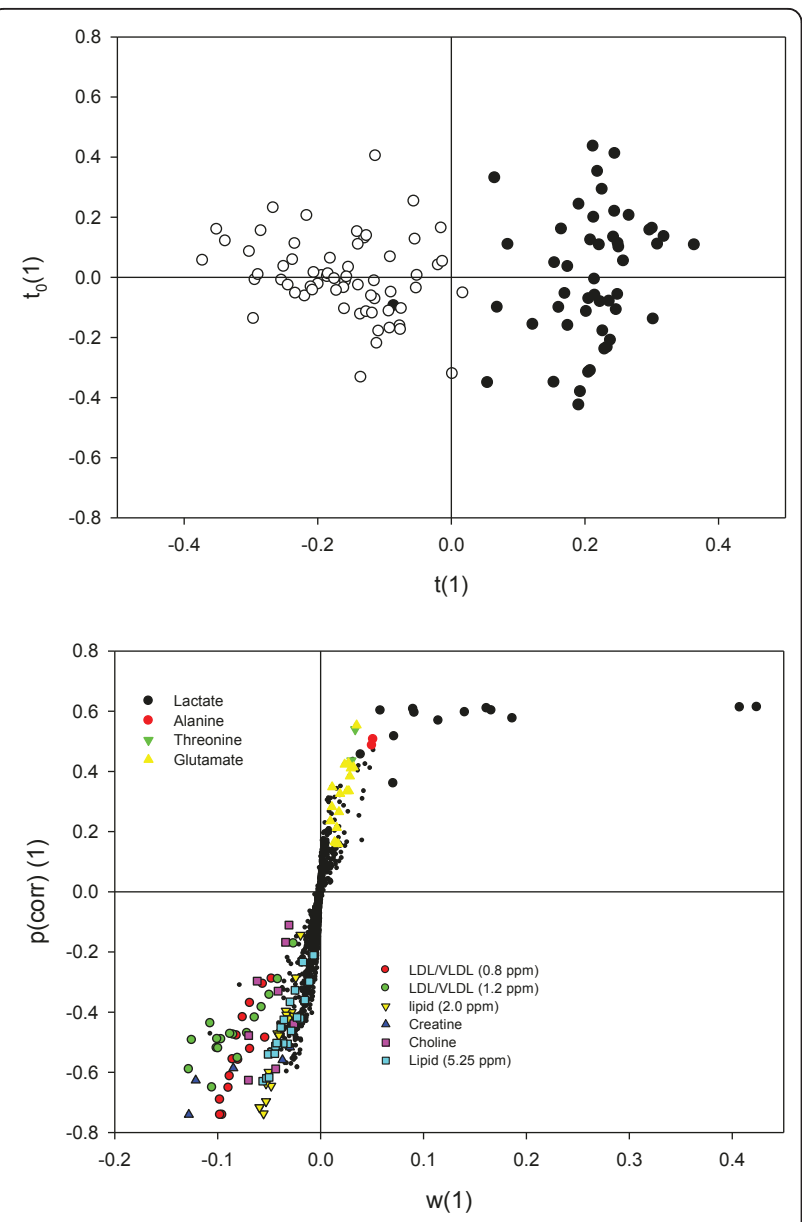

Figure 5 Plasma O-PLS. O-PLS score plot of all plasma samples from cloned pigs (closed symbols) and control pigs (open symbols) (a) and S-plot for plasma metabolites (b). $R^{2} X=0.72, R^{2} Y=0.83, Q^{2}$ (cum) $=0.65$. Variables with high discriminatory ability are identified in the S-plot and metabolites related to these variables are enlarged and coded with colours according to the legend. For clarity other variables are shown as dots.

variance between cloned and control pigs were observed (bile conjugated cholate/chenodeoxy cholate, plasma valine, and urine hippurate), and could thus not support the hypothesis that cloned pigs are less variable than control pigs. The numbers of ratios above and below one were 10 and 14 respectively, after removing values that originated from the same compound (Table 3). Under the hypothesis that the variance in cloned and control animals is similar $(\mathrm{p}=0.5)$ this distribution of ratios was not found to be significantly different from 50:50 and indicated a random distribution of variances.

\section{Discussion}

Cloned animals are expected to be more homogenous than outbred lines and could therefore represent a good model for research purposes where a small inter-individual variation is desired. However, only sparse data are
Table 2 Relative areas of selected metabolites in plasma determined by NMR integrals

\begin{tabular}{llcccccc}
\hline Compound & Observed $\boldsymbol{\delta}, \mathbf{p p m}$ & \multicolumn{3}{c}{ Clone } & \multicolumn{4}{c}{ Control } \\
\hline Alanine & 1.48 & 7.7 & \pm & 0.5 & 6.8 & \pm & 0.2 \\
Choline & 3.21 & 16 & \pm & 1.2 & 19 & \pm & 2.0 \\
Creatine & 3.92 & 3.1 & \pm & 0.4 & 5.2 & \pm & 0.7 \\
Glutamate & 2.45 & 8.2 & \pm & 0.2 & 7.5 & \pm & 0.1 \\
Lactate & 4.11 & 26 & \pm & 4.7 & 15 & \pm & 2.0 \\
Lipid & 5.30 & 18.5 & \pm & 0.9 & 22 & \pm & 1.2 \\
VLDL/LDL & 0.85 & 64 & \pm & 4.6 & 77 & \pm & 3.5 \\
VLDL/LDL & 1.25 & 89 & \pm & 5.2 & 109 & \pm & 3.4 \\
\hline
\end{tabular}

Results are reported as mean \pm standard deviation. Cloned pigs $(n=5)$ and control pigs $(n=6)$. All metabolites were significantly different between the two groups with $p<0.001$.

available about the phenotypic variation of cloned pigs $[9,10]$. The present study for the first time reports a metabolomic phenotyping of cloned pigs. Using NMRbased metabolomics we have shown that the metabolite profile of plasma and bile, but not urine, differed for cloned pigs and normal outbred pigs. In fact, the bile and plasma concentrations of multiple metabolites differed suggesting that the cloned pigs had an altered metabolic phenotype compared with the control outbred pigs.

For plasma samples it was identified that lactate levels were higher in cloned pigs than in normal outbred pigs. Alanine and glutamate, which are both linked to the citrate cycle, were also found in higher concentrations in cloned pigs. The reason for an increased plasma lactate remains unknown. In a study on heifers, cloned animals were found to have higher oxidative metabolism than control animals as assessed by isocitrate dehydrogenase, cytochrome-c oxidase and beta-hydroxyacyl-CoA dehydrogenase activities in muscle biopsies [20]. However, the increased plasma lactate levels observed in this study indicate higher anaerobic metabolism. Consequently, the increased lactate levels in the cloned pigs are probably a consequence of another mechanism. Oocytes and embryos are inevitably exposed to oxidative stress generated by reactive oxygen species during in vitro culture [21], and this might have impact on stress sensitivity post-natally in the cloned pig. The higher plasma lactate levels might therefore reflect higher stress sensitivity in cloned pigs compared with normal pigs due to the in vitro cloning procedure.

Plasma lipid and lipoprotein signals were higher in the control group as compared to the cloned group, which may be related to differences in the regulation of lipoprotein circulation. Likewise creatine, which is involved in cellular energy production, was elevated in control pigs. Thus, several metabolites indicate that the response of cloned pigs and control pigs to a dietary intervention is not the same, and this could affect the applicability in relation to human nutrition. 
Table 3 Variances calculated for integrals from bile, plasma, and urine

\begin{tabular}{|c|c|c|c|c|c|c|}
\hline \multirow[b]{2}{*}{ Peak number } & \multirow[b]{2}{*}{ Biofluid } & \multirow[b]{2}{*}{ Observed $\delta, \mathrm{ppm}$} & \multirow[b]{2}{*}{ Signal range } & \multicolumn{2}{|c|}{ Variance } & \multirow[b]{2}{*}{ v clone/v contro } \\
\hline & & & & Clone & Control & \\
\hline 1 & Bile & 0.62 & $87-102$ & 5,98 & 39.1 & 0.15 \\
\hline 4 & Bile & 2.75 & $2.9-14.7$ & 1.60 & 0.89 & 1.80 \\
\hline 7 & Bile & 3.45 & $8.5-13.5$ & 0.60 & 5.82 & $0.048 \mathrm{a}$ \\
\hline \multirow[t]{2}{*}{15} & Bile & 7.80 & $13.8-18.0$ & 0.19 & 1.26 & 0.15 \\
\hline & Bile & 7.98 & $1.8-4.9$ & 0.57 & 2.15 & 0.26 \\
\hline 5 & Bile & 3.07 & $5.9-16.8$ & 7.03 & 12.5 & 0.56 \\
\hline \multirow[t]{3}{*}{6,12} & Bile & 4.30 & $3.7-8.5$ & 0.94 & 0.74 & 1.27 \\
\hline & & 4.42 & $0.5-3.8$ & 0.049 & 0.036 & 1.38 \\
\hline & & 3.25 & $12-53$ & 15.2 & 7.05 & 2.16 \\
\hline 23 & Plasma & $1.91(\mathrm{~s})$ & $0.005-0.05$ & $5.40 \mathrm{E}-5$ & 4.47E-5 & 1.21 \\
\hline 21 & Plasma & 1.47 (d) & $0.006-0.1$ & 0.00038 & 0.00037 & 1.02 \\
\hline 33 & Plasma & $4.04(s)$ & $0-0.03$ & $2.77 \mathrm{E}-5$ & $4.38 \mathrm{E}-5$ & 0.63 \\
\hline 20 & Plasma & $4.10(q)$ & $0.03-0.7$ & 0.013 & 0.017 & 0.78 \\
\hline 27 & Plasma & $2.36(s)$ & $0.003-0.04$ & 4.54E-5 & 3.37E-5 & 1.35 \\
\hline 34 & Plasma & 7.18 (d) & $0.003-0.03$ & $3.12 \mathrm{E}-5$ & $3.32 \mathrm{E}-5$ & 0.94 \\
\hline 18 & Plasma & $1.03(d)$ & $0.004-0.3$ & 0.0018 & 0.00038 & $4.58 a$ \\
\hline 21 & Urine & $1.48(d)$ & $0.82-7.44$ & 2.2 & 2.5 & 0.87 \\
\hline 47 & Urine & 5.39 (s) & $1.89-31.4$ & 112 & 53 & 2.11 \\
\hline \multirow[t]{2}{*}{33} & Urine & $3.04(\mathrm{~s})$ & $44-497$ & 2.4E4 & $6.5 \mathrm{E} 3$ & 3.75 \\
\hline & Urine & 4.05 (s) & $31-349$ & $1.2 \mathrm{E} 4$ & $3.2 \mathrm{E} 3$ & 3.69 \\
\hline \multirow[t]{4}{*}{45} & Urine & 7.84 (d) & $3.6-40$ & 138 & 61 & $2.26 a$ \\
\hline & & $7.55(t)$ & $3.7-47$ & 185 & 67 & $2.85 a$ \\
\hline & & $3.97(\mathrm{~m})$ & $8.0-87$ & 524 & 178 & $2.94 a$ \\
\hline & & $7.64(\mathrm{t})$ & $8.1-28$ & 64 & 19 & $3.44 a$ \\
\hline 36 & Urine & 0.94 (d) & $2.8-18$ & 23 & 14 & 1.61 \\
\hline 37 & Urine & $1.36(\mathrm{~s})$ & $2.9-11$ & 7.2 & 4.0 & 1.80 \\
\hline 44 & Urine & $7.37(\mathrm{~m})$ & $1.8-17$ & 5.7 & 30.1 & 0.19 \\
\hline \multirow[t]{2}{*}{46} & Urine & $7.23(\mathrm{~d})$ & $2.2-68$ & 547 & 181 & 3.02 \\
\hline & Urine & 7.06 (d) & $0.7-60$ & 514 & 146 & 3.52 \\
\hline 49 & Urine & 5.08 (d) & $0.0-22.1$ & 79 & 15 & 5.20 \\
\hline 48 & Urine & 5.78 & $78-643$ & $2.0 \mathrm{E} 4$ & $1.2 \mathrm{E} 4$ & 1.62 \\
\hline
\end{tabular}

Variances based on normalized urine spectra and raw bile and plasma spectra. For each metabolite the ratios between cloned pig variances and control pig variances are shown. Peak numbers refer to the legend in Figure 1

a, significant values.

Furthermore, differences in bile composition, which is also under genetic control, could affect lipid metabolism [22]. The relative content of bile acids and phospholipids affects the micelle surface and core composition in the small intestine, and thus probably has an impact on the absorption of dietary lipids [23]. Increasing amounts of phospholipids have also been shown to reduce the critical micelle concentration of bile salts, thus affecting the number of micelles $[24,25]$. In the present study high amounts of bile acids in the bile were associated with an increased content of lipids in plasma, though a direct causal connection could not be established. Studies with rodents have shown that an increase in plasma bile acids induced by diet, reduce liver VLDL secretion and prevent elevated serum triacylglycerol concentration
[26]. These findings seem opposite to our data and further work is needed to elucidate the physiological role of production and circulation of bile acids, which also appears to be important for weight regulation [27].

In order to elucidate the potential of a cloned pig model, it is also important to consider the inter-individual variation. Consequently, for all bio-fluids the variance of individual metabolites was calculated and compared with the variance for control outbred pigs. This analysis did not reveal a significant difference in variation between the two groups. Consequently, based on the present study it cannot be concluded that the inter-individual variation in the metabolic phenotype is smaller for cloned pigs compared with normal outbred pigs. While a standardized genotype does not seem to 
affect the inter-individual variation, the gut-microbiota is known to affect host phenotype [19]. In the present study no systematic effect of gut-microbiota was expected since all animals received the same diet, and no difference in the contents of metabolites originating from microbial fermentation (e.g. acetic acid, butyric acid) could be demonstrated in the present study. Fluctuations in the host metabolome could arise, however, as a result of the complex interactions between nutrition, immune function, and gut-microbiota, and therefore the gut-microbiomes of cloned and normal pigs are currently being analyzed and will be the subject of additional papers. Therefore, when a low inter-individual variation between subjects is required, a standardization of the gut-microbiota might be more important than standardization of the genotype [28]. However, the present study only included a limited number of animals, and further studies with larger numbers of subjects are needed to substantiate these results. In addition the metabolites included in the analysis were chosen among the most intense resonances, which may introduce a bias. Less abundant metabolites, which are not easily detected by NMR-based metabolomics, are currently being analyzed by LC-MS-based metabolomics and will be the subject of a subsequent paper

\section{Conclusions}

In conclusion, despite the limited number of animals, the present metabolomic study on multiple bio-fluids clearly indicated alterations in the metabolic phenotype of cloned pigs compared with control pigs, and this should be taken into consideration when cloned animals are used as model animals. In addition, the present NMR-based analyses of plasma, bile and urine could not demonstrate a smaller inter-individual variation in cloned pigs compared with control pigs.

\section{Methods}

\section{Animals and sampling}

All experimental procedures involving animals were approved by the Danish Animal Experimental Committee.

Cloning was performed using somatic cell nuclear transfer as previously described [29] with donor cells from cultured ear fibroblasts obtained from a Danish Landrace $\times$ Yorkshire $(65 \%: 35 \%)$ sow. The cloned embryos were transferred surgically to surrogate sows (recipients) five to six days after cloning as described [30]. The cloned piglets were obtained by Caesarian section on gestation day 116 [30]. The sows were treated $24 \mathrm{~h}$ before with a prostaglandin analogue $(175 \mu \mathrm{g}$ Estrumate i.m., Pitman-Moore, UK).

As controls, normal litters (75\% Danish Landrace $\times$ 25\% Yorkshire) were obtained after standard artificial insemination and Caesarian section. All pigs were reared in the experimental stables of Aarhus University (Tjele, Denmark).

Two surrogate sows gave birth to 9 cloned piglets, of which 5 survived. Two normal control litters resulted in 18 female pigs, of which 6 were allocated as controls. Pigs were then nursed by surrogate sows and weaned after 28 days. They were kept on a standard diet for an additional 2 months, and were individually housed thereafter. The weight at 3 months of age was for clones $37.8 \pm 4.0 \mathrm{~kg}$ (Mean \pm SEM, $\mathrm{n}=5$ ) and for controls $37.9 \pm 2.3 \mathrm{~kg}(\mathrm{n}=6)$. The pigs were then fed ad libitum with a wheat-based high energy diet containing $10 \%$ sugar and $10 \%$ soy oil. Blood from the jugular vein was taken biweekly after overnight fasting for plasma preparation. The blood samples were stored on ice and centrifuged within $1.5 \mathrm{~h}$ at $3000 \mathrm{rpm}$ for $10 \mathrm{~min}$ at $4^{\circ} \mathrm{C}$. Pigs were killed with a bolt pistol at an age of $81 / 2$ months after overnight fasting. Weights of clones and controls at time of slaughter were $143.6 \pm 8.8 \mathrm{~kg}$ and $179.5 \pm 4.0 \mathrm{~kg}$, respectively. Blood samples were obtained during desangiunation for serum preparation, and urine and bile samples were obtained directly by puncture of the respective bladders. The blood samples were placed $1 \mathrm{~h}$ at room temperature and subsequently centrifuged at $3000 \mathrm{rpm}$ for $10 \mathrm{~min}$ at $4^{\circ} \mathrm{C}$. Serum, plasma, bile, and urine samples were frozen and kept at $-80^{\circ} \mathrm{C}$ until analysis.

\section{NMR spectroscopy}

The NMR measurements were performed at $310 \mathrm{~K}$ on a Bruker Avance III 600 spectrometer, operating at a ${ }^{1} \mathrm{H}$ frequency of $600.13 \mathrm{MHz}$, and equipped with a $5-\mathrm{mm}$ ${ }^{1} \mathrm{H}$ TXI probe (Bruker BioSpin, Rheinstetten, Germany).

For plasma and serum samples $500 \mu \mathrm{L}$ aliquots were mixed with $100 \mu \mathrm{D} \mathrm{D}_{2} \mathrm{O}$ containing $0.05 \% \mathrm{w} / \mathrm{w}$ sodium trimethylsilyl-[2,2,3,3- $\left.{ }^{2} \mathrm{H}_{4}\right]-1$-propionate (TMSP), for urine samples $300 \mu \mathrm{L}$ aliquots were mixed with $300 \mu \mathrm{L}$ $\mathrm{D}_{2} \mathrm{O}$ containing $0.005 \% \mathrm{w} / \mathrm{w}$ TMSP, while for bile samples $400 \mu \mathrm{L}$ aliquots were mixed with $200 \mu \mathrm{D}_{2} \mathrm{O}$ containing $0.025 \% \mathrm{w} / \mathrm{w}$ TMSP. Urine $\mathrm{pH}$ was adjusted to 7.0 prior to NMR measurements. On all bio-fluids, standard one-dimensional (1D) ${ }^{1} \mathrm{H}$ NMR spectra were acquired using single $90^{\circ}$ pulse experiment with a total of 64 scans and a relaxation decay of $5 \mathrm{~s}$. Water suppression was achieved by irradiating the water peak during the relaxation delay, and $16 \mathrm{~K}$ data points spanning a spectral width of $12.15 \mathrm{ppm}$ were collected. In addition, on plasma samples $1 \mathrm{D}{ }^{1} \mathrm{H}$ NMR spectra were also acquired with a Carr-Purcell-Meiboom-Gill (CPMG) delay added in order to attenuate broad signals from high-molecular-weight components. In the CPMG experiment a relaxation decay of $3 \mathrm{~s}$ was applied and 32 $\mathrm{K}$ data points spanning a spectral width of $17.36 \mathrm{ppm}$ were collected. 


\section{Data pre-processing}

All spectra were referenced to the TMSP signal at 0 ppm and the spectral region from 0.5 - 9.5 ppm was used. For multivariate data analysis spectra were aligned using the icoshift procedure [31] in MATLAB (version R2009b, The Mathworks Inc., Natick, MA, USA). Then spectra were subdivided into $0.006 \mathrm{ppm}$ spectral regions and integrated, leaving out the region 5.0 - $4.6 \mathrm{ppm}$, which included residual water resonance.

Normalization is often a prerequisite for bio-fluid analysis, especially for urine, because absolute urine metabolite concentrations are highly variable. However, the normalization procedure also affects score- and loading plots in multivariate data analysis [32]. In the present study multivariate data analysis (SIMCA-P+ software, Umetrics AB, Umeå, Sweden) of spectral data was carried out both on raw data and data normalized to the total signal intensities of the NMR spectra.

\section{Multivariate dataanalysis}

All multivariate analyses were carried out with full cross-validation (leave-one-out) and principal component analysis (PCA) was applied to the centered and paretoscaled data to explore any clustering behaviour of the samples. Furthermore, orthogonal partial least squares discriminant analysis (O-PLS-DA) was performed on spectra of the plasma samples.

Serum samples collected on the day of slaughter were included in the analysis of plasma samples. No grouping of the serum samples was observed in the PCA, and therefore plasma and serum was pooled for the multivariate analysis.

\section{Statistical analysis}

All statistical analyses were performed using the Statistics Toolbox in MATLAB. The relative integrals from plasma analysis were analysed using a one-way analysis of covariance.

For investigation of the variability of metabolite concentrations within groups, integrals of baseline separated metabolites were determined using Topspin 2.1 (Bruker Biospin, Faellanden, Switzerland). The variances of the integrals were determined within each experimental group (clone/control). For urine, normalized integrals were used, whereas for serum and bile absolute integrals were used. In order to test the equality of variance, an F-test was carried out using the vartest 2 function in Matlab, and p-values below 0.05 were considered significant.

For each metabolite the ratio between cloned and control variances was calculated. Values smaller than one thus reflect a lower variance in the cloned group and a value higher than one reflects a higher variance in the cloned group. For each metabolite the probability of the ratio to be either above or below one was expected to be 0.5 and binomially distributed. Thus, the probability of observing a number of ratios below one was computed using the binocdf function and a p-value below 0.05 was considered significant.

\section{Additional material}

Additional file 1: Compound IDs. Manuscript compound IDs,

compound names and Pubchem compound IDs.

Additional file 2: Raw data. All raw data as phased, baseline corrected, normalized, and binned NMR spectra.

Additional file 3: Integrals. Metabolite integrals for all individuals and Fstatistics.

\section{Acknowledgements}

Nina Eggers and Ditte Bruun Ditlev are acknowledged for their assistance with NMR measurements. Anette Pedersen, Ruth Kristensen, Janne Adamsen, and Klaus Villemoes are acknowledged for excellent technical work with cloning. Bente Synnesvedt is acknowledged for expert assistance with the surgeries. Dr. Henry Jørgensen is thanked for designing composition of the pig diet.

The project was financially supported by a grant from the Danish Strategic Research Council (FØSU 2101-06-0034), the Danish Advanced Technology Foundation (013-2006-2), and The Danish Research Council FTP (09-6649307).

\section{Author details}

'Department of Food Science, Science and Technology, Aarhus University, Aarslev, Denmark. ${ }^{2}$ Department of Animal Health and Bioscience, Science and Technology, Aarhus University, Tjele, Denmark. ${ }^{3}$ Department of Genetics and Biotechnology, Science and Technology, Aarhus University, Tjele, Denmark. ${ }^{4}$ Veterinary Reproduction and Obstetrics, Faculty of Life Sciences, University of Copenhagen, Frederiksberg C, Denmark. ${ }^{5}$ Department of Food Science, Science and Technology, Aarhus University, Tjele, Denmark.

\section{Authors' contributions}

JSG, HC, HCB and MSH designed the experiments. $\mathrm{YL}$ and $\mathrm{HC}$ cloned the embryos based on cells prepared by SP. MS transferred the embryos and performed the Caesarian sections. JSG, MSH, and KLC performed the animal experiment and the sampling, MRC did the NMR data analysis and assignments, statistical and multivariate data analysis and wrote the manuscript. MRC, HCB, JSG, and HC edited the manuscript, and the manuscript was read and approved by all authors.

Received: 3 May 2011 Accepted: 22 August 2011

Published: 22 August 2011

\section{References}

1. Guilloteau P, Zabielski R, Hammon HM, Metges CC: Nutritional programming of gastrointestinal tract development. Is the pig a good model for man? Nutr Res Rev 2010, 23:4-22.

2. Litten-Brown JC, Corson AM, Clarke L: Porcine models for the metabolic syndrome, digestive and bone disorders: a general overview. Animal 2010, 4:899-920.

3. Bell FP, Gerrity RG: Evidence for an altered lipid metabolic state in circulating blood monocytes under conditions of hyperlipemia in swine and its implications in arterial lipid metabolism. Arteriosd Throm Vas Biol 1992, 12:155.

4. Dixon JL, Stoops JD, Parker JL, Laughlin MH, Weisman GA, Sturek M: Dyslipidemia and vascular dysfunction in diabetic pigs fed an atherogenic diet. Arteriosd Throm Vas Biol 1999, 19:2981.

5. Mahley RW, Weisgraber KH, Innerarity T, Brewer HB Jr, Assmann G: Swine lipoproteins and atherosclerosis. Changes in the plasma lipoproteins and apoproteins induced by cholesterol feeding. Biochemistry 1975 , 14:2817-2823. 
6. Terpstra AHM, Lapre JA, de Vries HT, Beynen AC: Transiency of the different cholesterolaemic responses to dietary cellulose and psyllium in pigs and two strains of hamsters. J Anim Physiol Anim Nutr 2000, 84:178-191.

7. Jensen TW, Mazur MJ, Pettigew JE, Perez-Mendoza VG, Zachary J, Schook LB: A Cloned Pig Model for Examining Atherosclerosis Induced by High Fat, High Cholesterol Diets. Anim Biotechnol 2010, 21:179-187.

8. St.John J: Aberrant heteroplasmic transmission of mtDNA in cloned pigs arising from double nuclear transfer. Mol Reprod Dev 2005, 72:450-460.

9. Archer GS, Dindot S, Friend TH, Walker S, Zaunbrecher G, Lawhorn B, Piedrahita JA: Hierarchical phenotypic and epigenetic variation in cloned swine. Biol Reprod 2003, 69:430

10. Carter DB, Lai L, Park KW, Samuel M, Lattimer JC, Jordan KR, Estes DM, Besch-Williford C, Prather RS: Phenotyping of transgenic cloned piglets. Cloning Stem Cells 2002, 4:131-145.

11. Rezzi S, Ramadan Z, Martin FPJ, Fay LB, van Bladeren P, Lindon JC, Nicholson JK, Kochhar S: Human metabolic phenotypes link directly to specific dietary preferences in healthy individuals. J Proteome Res 2007, 6:4469-4477

12. Bertram HC, Malmendal A, Nielsen NC, Straadt IK, Larsen T, Bach Knudsen KE, Nygaard Lærke H: NMR based metabonomics reveals that plasma betaine increases upon intake of high fiber rye buns in hypercholesterolemic pigs. Mol Nutr Food Res 2009, 53:1055-1062.

13. Bertram HC, Knudsen KEB, Serena A, Malmendal A, Nielsen NC, Frette XC, Andersen HJ: NMR-based metabonomic studies reveal changes in the biochemical profile of plasma and urine from pigs fed high-fibre rye bread. Br J Nutr 2006, 95:955-962

14. He Q, Kong X, Wu G, Ren P, Tang H, Hao F, Huang R, Li T, Tan B, Li P: Metabolomic analysis of the response of growing pigs to dietary Larginine supplementation. Amino Acids 2009, 37:199-208

15. Nissen PM, Nebel C, Oksbjerg N, Bertram HC: Metabolomics Reveals Relationship between Plasma Inositols and Birth Weight: Possible Markers for Fetal Programming of Type 2 Diabetes. J Biomed Biotechnol 2011.

16. Lindon JC, Nicholson JK, Everett JR: NMR spectroscopy of biofluids. Annu Rep NMR Spectrosc 1999, 38:1-88.

17. Wishart DS, Tzur D, Knox C, Eisner R, Guo AC, Young N, Cheng D, Jewell K, Arndt D, Sawhney S, Fung C, Nikolai L, Lewis M, Coutouly M-A, Forsythe I, Tang P, Shrivastava S, Jeroncic K, Stothard P, Amegbey G, Block D, Hau DD, Wagner J, Miniaci J, Clements M, Gebremedhin M, Guo N, Zhang Y, Duggan GE, MacInnis GD, Weljie AM, Dowlatabadi R, Bamforth F, Clive D, Greiner R, Li L, Marrie T, Sykes BD, Vogel HJ, Querengesser L: HMDB: the Human Metabolome Database. Nucleic Acids Res 2007, 35:D521-D526.

18. Gowda GAN, Somashekar BS, ljare OB, Sharma A, Kapoor VK, Khetrapal CL: One-step analysis of major bile components in human bile using $\mathrm{H}-1$ NMR spectroscopy. Lipids 2006, 41:577-589.

19. Claus SP, Tsang TM, Wang Y, Cloarec O, Skordi E, Martin FP, Rezzi S, Ross A, Kochhar S, Holmes E, Nicholson JK: Systemic multicompartmental effects of the gut microbiome on mouse metabolic phenotypes. Mol Syst Biol 2008, 4.

20. Jurie C, Picard B, Heyman Y, Cassar-Malek I, Chavatte-Palmer P, Richard C, Hocquette JF: Comparison of cloned and non-cloned Holstein heifers in muscle contractile and metabolic characteristics. Animal 2009, 3:244-250.

21. You J, Kim J, Lim J, Lee E: Anthocyanin stimulates in vitro development of cloned pig embryos by increasing the intracellular glutathione level and inhibiting reactive oxygen species. Theriogenology 2010, 74:777-785.

22. Norlin M, Wikvall K: Enzymes in the conversion of cholesterol into bile acids. Curr Mol Med 2007, 7:199-218.

23. Miller KW, Small DM: The phase behavior of triolein, cholesterol, and lecithin emulsions. J Colloid Interface Sci 1982, 89:466-478.

24. Naylor LJ, Bakatselou V, Dressman JB: Comparison of the Mechanism of Dissolution of Hydrocortisone in Simple and Mixed Micelle Systems. Pharm Res 1993, 10:865-870.

25. Carey MC, Small DM: Micelle Formation by Bile Salts: Physical-Chemical and Thermodynamic Considerations. Arch Intern Med 1972, 130:506-527.

26. Watanabe M, Houten SM, Wang L, Moschetta A, Mangelsdorf DJ, Heyman RA, Moore DD, Auwerx J: Bile acids lower triglyceride levels via a pathway involving FXR, SHP, and SREBP-1c. J Clin Invest 2004, 113:1408-1418.

27. Liaset B, Madsen L, Hao Q, Criales G, Mellgren G, Marschall HU, Hallenborg P, Espe M, Frøyland L, Kristiansen K: Fish protein hydrolysate elevates plasma bile acids and reduces visceral adipose tissue mass in rats. Biochim Biophys Acta, Mol Cell Biol Lipids 2009, 1791:254-262.

28. Kau AL, Ahern PP, Griffin NW, Goodman AL, Gordon Jl: Human nutrition, the gut microbiome and the immune system. Nature 2011, 474:327-336.

29. Kragh PM, Vaita G, Corydon TJ, Purup S, Bolund L, Callesen H: Production of transgenic porcine blastocysts by hand-made cloning. Reproduction Fertility and Development 2004, 16:315-318.

30. Schmidt M, Kragh PM, Li J, Du Y, Lin L, Liu Y, Bogh IB, Winther KD, Vajta G, Callesen $\mathrm{H}$ : Pregnancies and piglets from large white sow recipients after two transfer methods of cloned and transgenic embryos of different pig breeds. Theriogenology 2010, 74:1233-1240.

31. Savorani F, Tomasi G, Engelsen SB: icoshift: A versatile tool for the rapid alignment of 1D NMR spectra. J Magn Reson 2010, 202:190-202.

32. Zhang S, Zheng C, Lanza IR, Nair KS, Raftery D, Vitek O: Interdependence of Signal Processing and Analysis of Urine 1H NMR Spectra for Metabolic Profiling. Anal Chem 2009, 81:6080-6088.

doi:10.1186/1472-6793-11-14

Cite this article as: Clausen et al: Metabolomic phenotyping of a cloned pig model. BMC Physiology 2011 11:14.

\section{Submit your next manuscript to BioMed Central and take full advantage of:}

- Convenient online submission

- Thorough peer review

- No space constraints or color figure charges

- Immediate publication on acceptance

- Inclusion in PubMed, CAS, Scopus and Google Scholar

- Research which is freely available for redistribution

Submit your manuscript at www.biomedcentral.com/submit
C Biomed Central 